\title{
ON THE DYNAMICAL PROCESS OF THE PARKFIELD EARTHQUAKE OF JUNE 28, 1966
}

\author{
Ichiro KAWASAKI \\ Geophysical Institute, Faculty of Science, \\ University of Tokyo, Tokyo, Japan \\ (Received August 2, 1974; Revised January 10, 1975)
}

The seismic acceleration recorded at the Parkfield, Cholame No. 2 station in California at the time of the Parkfield earthquake of June 28, 1966, is analysed to determine the focal parameters on the dynamical process of faulting.

A direct comparison of the calculated accelerations with the recorded one yields focal parameter estimates of $0.5-0.7 \mathrm{sec}$ for the rise time of the source time function, $50-70 \mathrm{~cm}$ for the average dislocation and $50 \mathrm{~cm} / \mathrm{sec}$ for the particle velocity of the fault motion. The effective stress is determined from the particle velocity to be $25-50$ bars by the dynamic faulting models recently developed. The wave form of the calculated acceleration shows a good peak-to-peak correspondence with the recorded one, only when the source time function is assumed to be of a ramp type. This suggests that the ramp function is a good approximation for the source time function of this earthquake.

We obtain an amplitude of about $7 \mathrm{~cm}$ for the perpendicular component of the theoretical ground displacement if the values of $50-70 \mathrm{~cm}$ for the average dislocation and of $0.5-0.7 \mathrm{sec}$ for the rise time as estimated above are used. The value of $30 \mathrm{~cm}$ for the amplitude of displacement, obtained by previous investigators from numerical integration of the record acceleration, seems to be too large. The integrated displacements may not be good approximations for the true ground displacements.

\section{Introduction}

The investigation of the focal process from the data in the near-field has become more and more important because full data are required for a better understanding of the dynamic process of faulting, in view of the recent advances in seismology.

Maruyama (1963) and Haskell $(1964,1969)$ gave mathematical expressions of seismic waves due to a shear fault located in an infinite homogeneous medium. AKI (1968, 1972), KANAMORI (1971, 1972) and Mrkumo (1973) demonstrated the applicability of the theoretical seismograms calculated from 
formulae of Maruyama or Haskell in the focal-mechanism studies using the near-field data. These investigators discussed the focal parameters assuming that, doubling the displacements for an infinite medium, they obtain good approximations for the free surface displacements for a semi-infinite medium. However, the validity of this assumption of the doubling of the displacement for the free surface effects was not evident.

KAWASAKI et al. $(1972,1973)$ applied the Cagniard method to the expressions given by SATO (1969), taking the free surface effects into account, and gave exact expressions of the seismic waves due to a double couple point source in a semi-infinite medium. They discussed the near-field characteristics of all phases in detail and pointed out some problems; for example, an SP wave refracted at the free surface has an unexpectedly large amplitude comparable to that of the $\mathbf{P}$ wave and cannot be neglected when discussing the focal mechanism. KAWASAKI and SUZUKI (1974) synthesized the seismograms due to a moving shear fault model in the semi-infinite and infinite media and compared them with the actual records of the Sanriku earthquake of 1933. They estimated the rise time of the source time function to be $5 \mathrm{sec}$ or less, based on the fact that the SP phase can be discriminated as a later phase from the initial $\mathbf{P}$ motion for a rise time shorter than $5 \mathrm{sec}$.

The Parkfield earthquake of June 28, 1966, has been studied by many investigators (AKr, 1968; Cloud and Perez, 1967; EATON, 1967; EA TON et al., 1970; Filson and McEvilly, 1967; Housner and Trifunac, 1967; Tsai and AKI, 1969). However, it has been recognized that an analysis on the basis of a direct comparison between the theoretical and recorded accelerations is very difficult, although it is expected to yield some information on the dynamical process of faulting. The purposes of the present work are to calculate the ground displacements for the semi-infinite and the infinite media and to compare them with the numerical results obtained by AKI $(1968,1972)$, with particular attention to the free surface effects on the wave forms of the seismograms. Another purpose is to determine the focal parameters on the dynamical process of faulting, on the basis of direct comparison of the theoretical accelerations with the recorded one. SATO (1973) derived mathematical expressions, in a form pertinent for numerical calculation, of the seismic waves due to a shear fault in a multi-layered medium by the Cagniard-de Hoop technique. However, since these expressions for the multi-layered medium require a large amount of numerical computation, we do not refer to them in the present study. We lack complete mathematical models and sufficient data to determine the effective stress precisely. We have used approximate faulting models recently proposed by BRUNE (1970), KANAMORI (1972) and IDA and AKI (1972) in order to determine the order of magnitude of the effective stress which generated this earthquake. 


\section{Fault Geometry}

To construct a fault geometry for the Parkfield earthquake, we first summarize the results of the source studies obtained by previous investigators. BROWN and VEDDER (1967) inferred the fault length $L$ to be $30-40 \mathrm{~km}$. EATON (1967) estimated the rupture velocity $v_{c}$ to be $2.2 \mathrm{~km} / \mathrm{sec}$. FILson and McEvILLY (1967) determined $L=30 \mathrm{~km}, v_{c}=2.2 \mathrm{~km} / \mathrm{sec}$ from a spectral analysis of Love wave. EATON et al. (1970) supposed the vertical extension of the fault to be $10 \mathrm{~km}$, based on the aftershock distribution as shown in Fig. 1. TSAI and AKI (1969) estimated the seismic moment to be $1.4 \times 10^{25} \mathrm{dyne} \cdot \mathrm{cm}$ from the spectral analysis of long period surface waves. ScHolz et al. (1969) inferred the dislocation distribution to be $30 \mathrm{~cm}$ over the fault from $4 \mathrm{~km}$ to $10 \mathrm{~km}$ in depth from the geodetic and surface wave data.

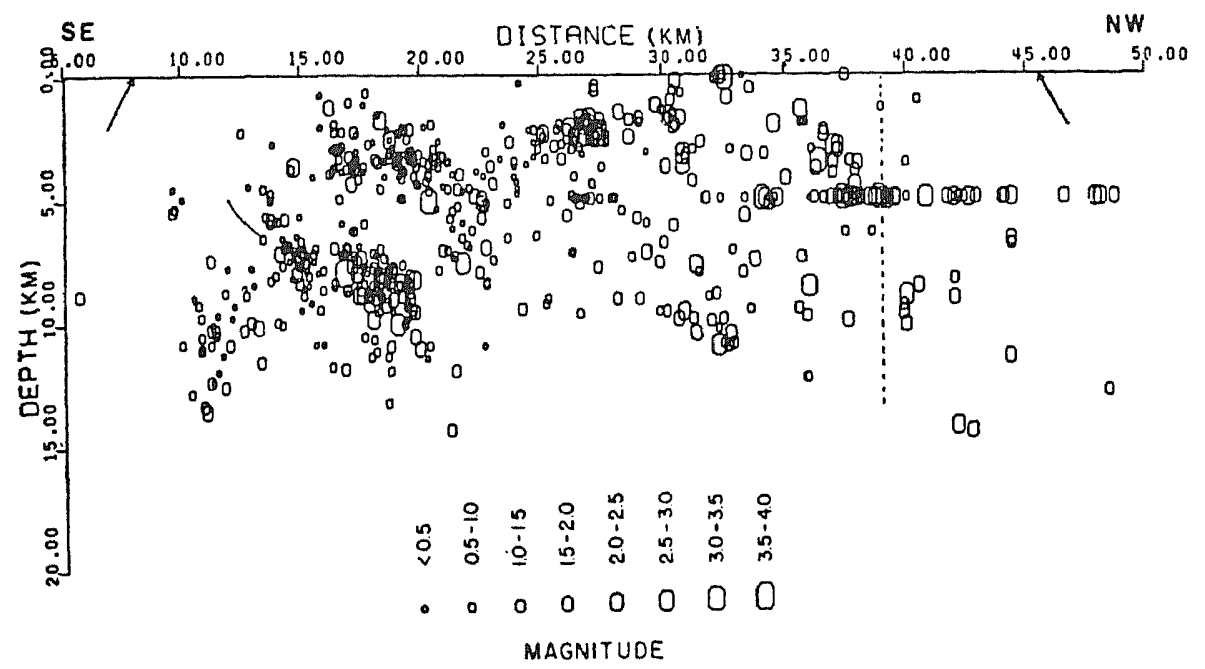

Fig. 1. Aftershock distribution on the reference fault plane. (Reproduced from a paper by EATON et al. (1970)).

Following the above-mentioned investigations, the focal parameters are assumed in the present study as follows:

$$
\begin{array}{ll}
\delta=90^{\circ} & : \text { dip angle (vertical fault), } \\
\lambda=-180^{\circ} & : \text { slip direction (right lateral), } \\
v_{\mathrm{P}}=6.0 \mathrm{~km} / \mathrm{sec} & : \text { P wave velocity, } \\
v_{\mathrm{S}}=3.5 \mathrm{~km} / \mathrm{sec} & : \text { S wave velocity, } \\
v_{\mathrm{c}}=2.2 \mathrm{~km} / \mathrm{sec} & : \text { rupture velocity, } \\
\mu=2.0 \times 10^{11} \text { dyne.cm } & : \text { rigidity, } \\
L=30 \mathrm{~km} & : \text { fault length, } \\
W=10 \mathrm{~km} & : \text { fault width. }
\end{array}
$$




\section{The Recorded Acceleration and the Integrated Displacement}

At the time of the Parkfield earthquake, the perpendicular (to the fault)

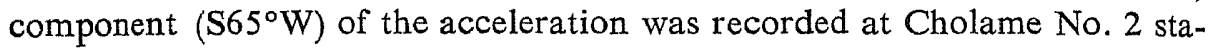
tion at $80 \mathrm{~m}$ from the fault, while an accelerograph of the parallel component $\left(\mathrm{S} 25^{\circ} \mathrm{E}\right)$ was unfortunately inoperative. The characteristic period of the functioning accelerograph is 0.064 second, magnification 1.0 and damping coefficient 0.57 . Because the characteristic period of the accelerograph is very short, the seismic motions recorded in the period range greater than about 0.1 second are approximately proportional to the ground accelerations. The full record is reproduced in the papers of Cloud and Perez (1967) and Housner and Trifunac (1967). The component dealt with in the following is this perpendicular one except when otherwise specified.

HousNer and TrifunAC (1967) and AKI $(1968,1972)$ obtained the integrated ground displacement from the recorded acceleration. The integrated ground displacement had the duration of about $1.5 \mathrm{sec}$ and a maximum amplitude of about $30 \mathrm{~cm}$ as shown in Fig. 2. This is tentatively considered as "the observed ground displacement" in the next section, where a comparison between the observed displacement and theoretical one is made.

AKI (1968, 1972), further, calculated the theoretical ground displacements by integration of the formulae given by MARuYama (1963). From comparison between "the observed" and the theoretical ground displacements,

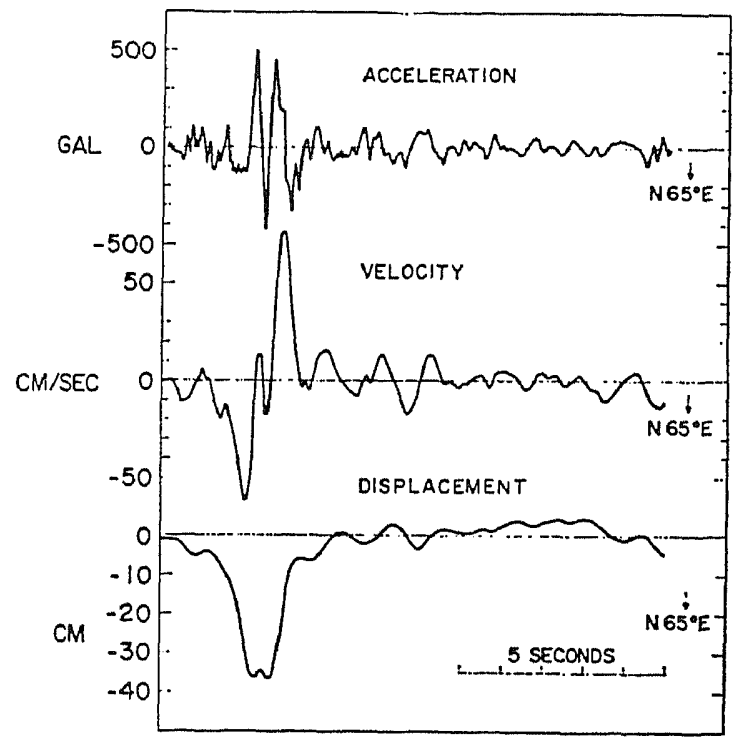

Fig. 2. Acceleration of perpendicular component and its velocity and displacement integrated from the recorded acceleration. (Reproduced from a paper by AKr (1972)). 
he determined the average dislocation over a fault to be $60-90 \mathrm{~cm}$, the rise time $0.4-0.9 \mathrm{sec}$, and the stress drop $20-100$ bars and $\eta \bar{\sigma} 2$ bars, where $\eta$ is the efficiency of seismic radiation and $\bar{\sigma}$ is the average of initial stress $\sigma_{0}$ and final stress $\sigma_{1}$ along the fault.

In this calculation, AKI (1968) used a fault segment as shown in Fig. 3. The fault length and the fault width are taken to be $2 \mathrm{~km}$ and $3 \mathrm{~km}$, respec-

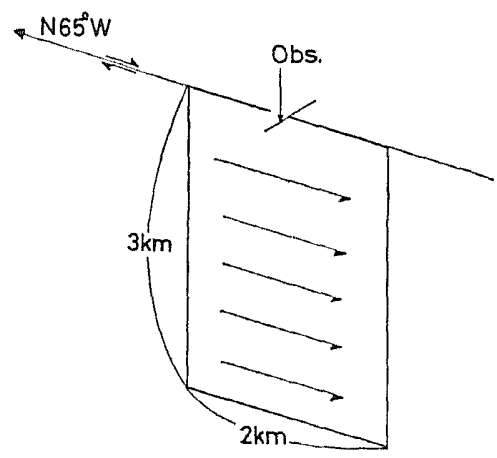

Fig. 3. A fault segment used in the calculation of theoretical seismograms. Fault length is $2 \mathrm{~km}$; fault width, $3 \mathrm{~km}$. Rupture is assumed to propagate unilaterally from the north to the south with a constant velocity $v_{c}=2.2 \mathrm{~km} / \mathrm{sec}$.

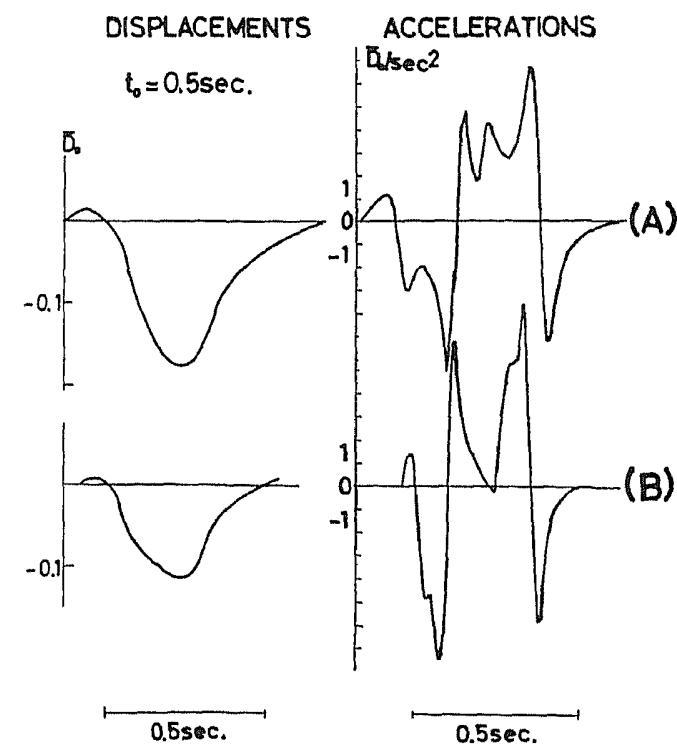

Fig. 4. The theoretical ground displacements and accelerations for the infinite medium. (A): fault length $4 \mathrm{~km}$. (B): fault length $2 \mathrm{~km}$. 
tively. Evaluating the distance dependence of seismic spectra, he proved that the contribution from the other fault segments is small, and that the effects of the fault width on the seismic spectrum is negligible at this short distance. In Fig. 4, the theoretical ground displacement and acceleration of the perpendicular component for an infinite medium with a fault length of $4 \mathrm{~km}$ are compared to those with a fault length of $2 \mathrm{~km}$. From this figure, we find that the contribution to the perpendicular component from the segments other than that of Fig. 3 may be small and may not alter the dislocation and rise time estimates in the following sections to a large extent.

\section{Theoretical Ground Displacements}

When we calculate theoretical ground displacements, the fault segment of the same dimension as Aki's in Fig. 3 (fault length $=2.0 \mathrm{~km}$, the vertical extension of the fault $=3.0 \mathrm{~km}$ ) is used for the purposes of direct comparison with Aki's results and for economy of computation time. A numerical computation for the semi-infinite medium requires a time as much as twenty or

\section{GROUND DISPLACEMENTS}
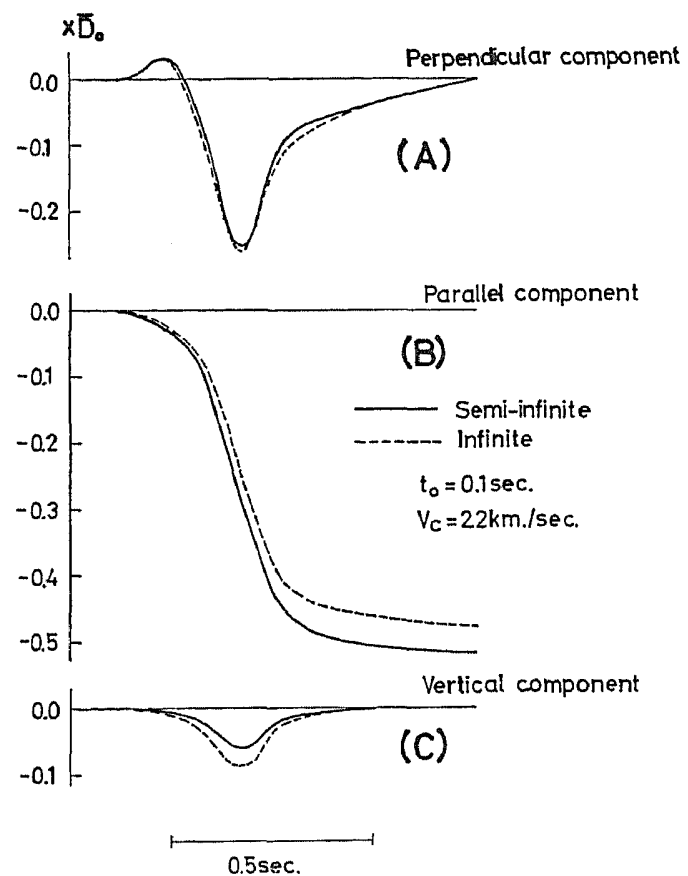

(C)

Fig. 5. Ground displacements of each component for a semiinfinite medium (solid) as compared with those for an infinite medium (broken). 
more times as that for the infinite medium. In the following, we assume that the results obtained for the above small segment hold for the faulting process of the other segment of the fault. For the numerical integration along the fault, the area of the fault element is taken to be very small when the element

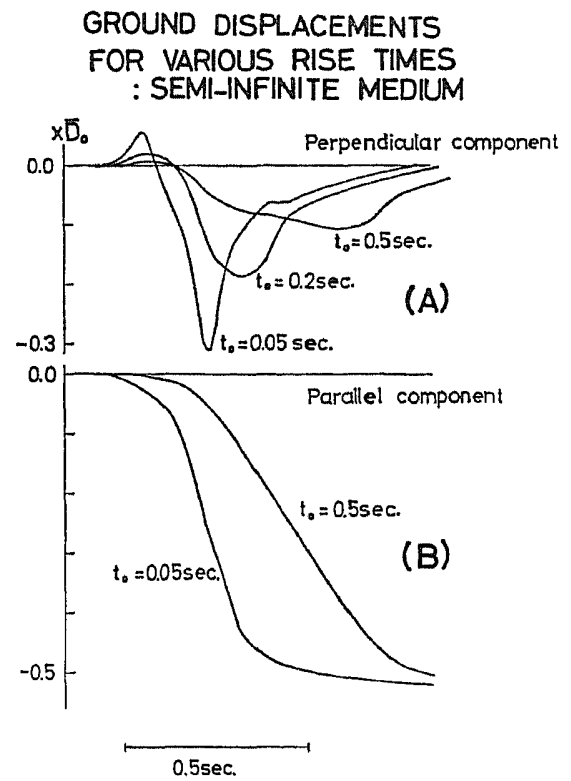

Fig. 6. The ground displacements for a semi-infinite medium for various rise times. (A): perpendicular component. (B): parallel component.

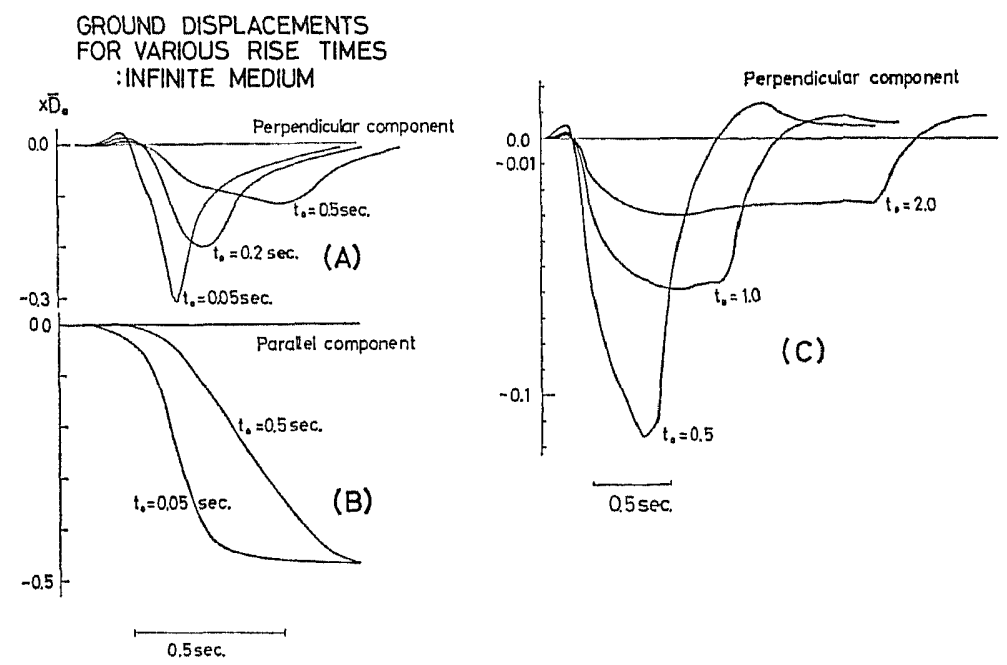

Fig. 7. The ground displacements for an infinite medium for various rise times. 
is located close to the station, in order to secure convergence of the numerical integration.

In Fig. 5, theoretical ground displacements for the semi-infinite medium (solid curves) and those for the infinite medium (broken curves) are illustrated. For the displacements for the infinite medium, the amplitudes are doubled as a simple correction of the free surface effects. Units of the amplitudes of the theoretical ground displacements and accelerations are $\bar{D}_{0}$ and $\bar{D}_{0} / \mathrm{sec}^{2}$, respectively, in this and the following figures. $\bar{D}_{0}$ is the average dislocation over the fault. The time increment in these seismograms is taken to be $0.1 / 6.0$ $(=0.0167) \mathrm{sec}$, small enough to be differentiable twice. As is seen in Fig. 5, the free surface effects are small and the doubling of the displacements for the infinite medium is an excellent correction for the free surface effects in this case, such as the fault motion of the vertical strike-slip type.

In Figs. 6 and 7 is illustrated how the wave forms change, as rise time $t_{0}$ varies. For example, when $t_{0}=0.5 \mathrm{sec}$, the maximum amplitude and the pulse width of the perpendicular component of the displacement are about $0.12 \vec{D}_{0}$ and $0.5 \mathrm{sec}$, while they are $0.06 \bar{D}_{0}$ and $1.0 \mathrm{sec}$ when $t_{0}=1.0 \mathrm{sec}$. AKr (1972) obtained a maximum amplitude of $(0.13-0.14) \bar{D}_{0}$ when $t_{0}=0.4 \mathrm{sec}$.

The ground displacement at this short distance may be approximately contributed from the shallower segment of the fault. Consequently, the estimated value of the dislocation from the above data probably indicates the maximum dislocation, $D_{\max }$, at the uppermost segment of the faulting area. A comparison of the calculated displacements with the "observed one" yields estimates of $0.5-1.0 \mathrm{sec}$ for the rise time $t_{0}$ and $250-500 \mathrm{~cm}$ for the maximum dislocation $D_{\max }$. From this, we obtain the average dislocation $\bar{D}_{0}$ over the fault to be $200-400 \mathrm{~cm}$ through the relation $\bar{D}_{0}=(\pi / 4) D_{\max }$ of KNOPOFF (1958). However, this value disagrees considerably much with the estimate of $\bar{D}_{0}=$ $15 \mathrm{~cm}$ or $30 \mathrm{~cm}$ derived from the surface wave data (TsAI and AKI, 1969) and geodetic data (Scholz et al., 1969).

\section{Theoretical Accelerations of the Ground Motion}

The theoretical accelerations are found by numerical differentiations of the ground displacements and are compared with the main part of the recorded acceleration shown in Fig. 8. In the following sections, a dot - over a letter denotes differentiation with respect to time. Only the theoretical accelerations for the semi-infinite medium are used unless specified for the infinite medium.

The removable strip method (LANCzos, 1956) is used to compute the second derivatives. The derivative at any time is found by the analytical differentiation of the parabolic curve determined by the least squares method 


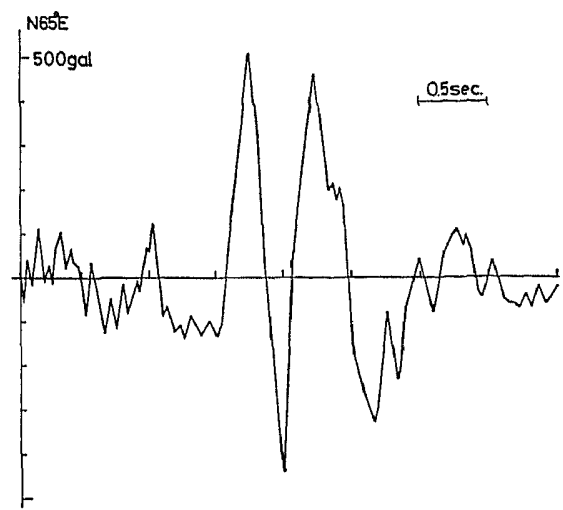

Fig. 8. Main part of the recorded acceleration.

from the 5-points. We repeat this again to obtain the second derivatives, i.e., accelerations.

Namely,

$$
\begin{aligned}
\ddot{u}(t)= & \frac{4 u(t+4 \Delta t)+4 u(t+3 \Delta t)+u(t+2 \Delta t)-4 u(t+\Delta t)-10 u(t)}{100(\Delta t)^{2}} \\
& +\frac{-4 u(t-\Delta t)+u(t-2 \Delta t)+4 u(t-3 \Delta t)+4 u(t-4 \Delta t)}{100(\Delta t)^{2}}
\end{aligned}
$$

Theoretical accelerations are exhibited in Fig. 9 for various rise times from $0.5 \mathrm{sec}$ to $0.7 \mathrm{sec}$. From the figure, a significant peak-to-peak correspondence of the theoretical accelerations with the recorded one (Fig. 8) is found, while wave forms do not show a good resemblance. The above correspondence yields an estimate of $0.5-0.7 \mathrm{sec}$ for the rise time. Since the maximum amplitude of the recorded acceleration is about 500 gal, we obtain $D_{\max }=65 \mathrm{~cm}$ for

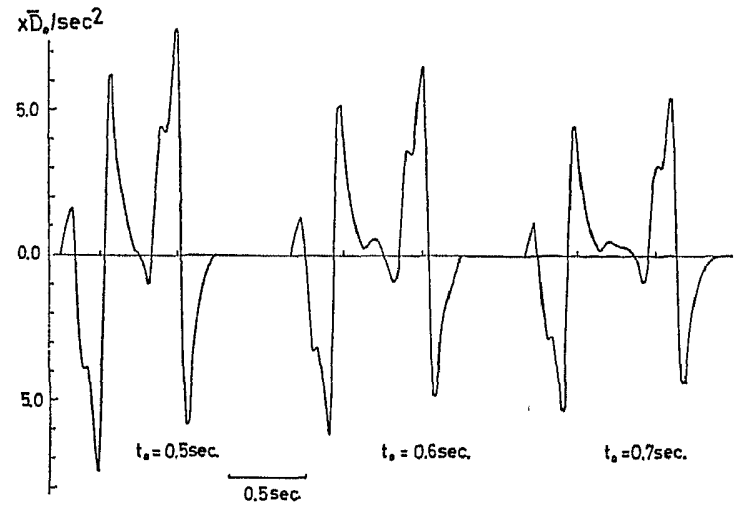

Fig. 9. Theoretical accelerations for the rise times $0.5,0.6$ and $0.7 \mathrm{sec}$. 
$t_{0}=0.5 \mathrm{sec}$ and $D_{\max }=90 \mathrm{~cm}$ for $t_{0}=0.7 \mathrm{sec}$. This yields an estimate of the average dislocation $\widetilde{D}_{0}=50-70 \mathrm{~cm}$. The particle velocity of the fault motion $\bar{D}_{0} / 2 t_{0}$ is evaluated to be $50 \mathrm{~cm} / \mathrm{sec}$ for the respective rise time estimates.

If a source time function of the ramp type is assumed, the maximum amplitude of the theoretical acceleration is approximately proportional to $\bar{D}_{0} / t_{0}$, as is seen in Fig. 9. Hence, when discussing the seismic acceleration near a fault, the rise time, which is strongly connected with the dynamical process of faulting, is very significant.

\section{Check of the Method of the Numerical Differentiation}

We check the error attributed to the numerical differentiation as shown in Fig. 10. The respective curves are the accelerations found by the following methods:

$$
\begin{aligned}
& \text { the removable strip method as described by the formula } \\
& \begin{array}{c}
\ddot{u}(t)=[u(t+\Delta t)+u(t-\Delta t)-2 u(t)] / 2(\Delta t)^{2} \\
\ddot{u}(t)=[2 u(t+2 \Delta t)-u(t+\Delta t)-2 u(t)-u(t-\Delta t)+2 u(t-2 \Delta t)] / 7(\Delta t)^{2} \\
\ddot{u}(t)=\frac{1}{2 \pi} \int_{-\infty}^{\infty}\left(-\omega^{2}\right)[\text { Filter }(\omega)] \mathrm{e}^{i \omega t} \mathrm{~d} \omega \int_{-\infty}^{\infty} u\left(t^{\prime}\right) \mathrm{e}^{-i \omega t^{\prime}} \mathrm{d} t^{\prime} \\
\text { Filter }(\omega)=\cos ^{2}\left(\frac{\omega}{60 \text { cycle }}\right) \quad \text { for }|\omega|<30 \pi \text { cycle, } \\
=0
\end{array}
\end{aligned}
$$

The formula (2) is the primitive method and causes much short-period noise. Formula (3) is the analytical second derivative of the parabolic curve determined by the least squares method from 5-points. Formula (4) is the Fourier transformation method. As is seen in Fig. 10, it is concluded that the numerical differentiation does not cause such substantial disturbance to the wave forms as to alter the estimate of $0.5-0.7 \mathrm{sec}$ for the rise time, while the values of the maximum amplitudes may contain error within a factor of 2 .

A variation of the wave form of the theoretical acceleration for various rupture models is examined in Fig. 11. The graph (A) is the same as shown in Fig. 9 for $t_{0}=0.5 \mathrm{sec}$. The graph (B) is the acceleration for a fault model, in which the rupture velocity has a downward component of $v_{c}=3 \mathrm{~km} / \mathrm{sec}$. The graphs of (C) and (D) are those for fault models, in which the top of the fault is located at $50 \mathrm{~m}$ and $100 \mathrm{~m}$ in depth, respectively. From the graphs of (C) and (D), it is found that the deeper the top of the fault is located, the smaller the maximum amplitude of the acceleration. Therefore, a fault model which has no dislocation distribution on a shallow portion of the fault as 


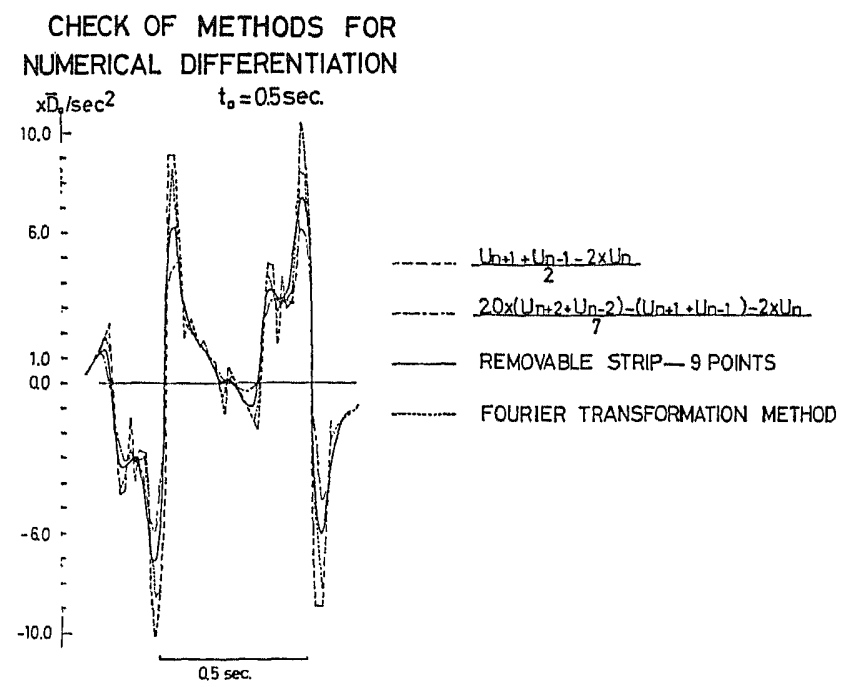

Fig. 10. Theoretical accelerations found by various methods of the numerical differentiation. Rise time is $0.5 \mathrm{sec}$.

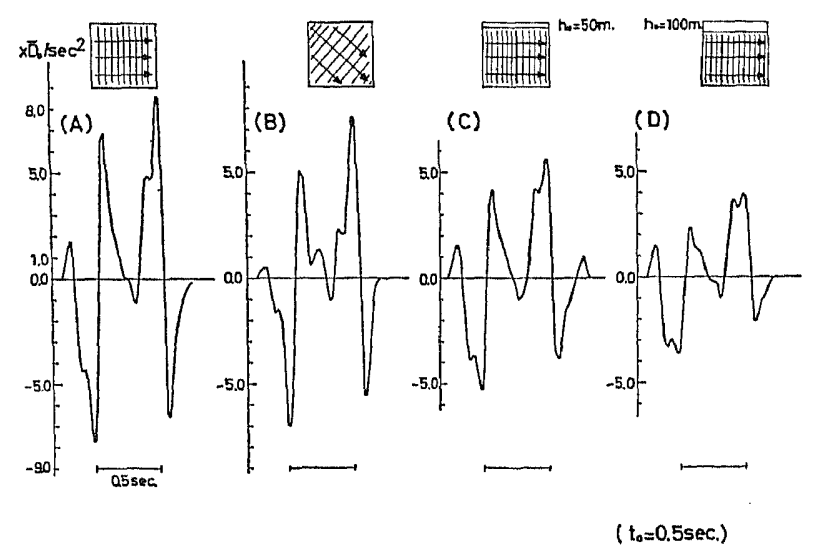

Fig. 11. Theoretical accelerations for various fault models.

presented by ScHoLz et al. (1969) will never explain the large amplitude of the recorded acceleration.

From the above comparisons and considerations, we can conclude that the error caused by the numerical differentiation and the faulting models can be neglected, except for the error of the maximum amplitude within a factor of 2 . 


\section{Source Time Function}

In Section 4, it is pointed out that, in the near-source region, the rise time plays a great role in seismic waves. This fact suggests a possibility of finding the type of the source time function, due to the comparison of the synthetic acceleration with the recorded one.

We denote the free surface displacement and acceleration by $u(t)$ and $\ddot{u}(t)$, when the source time function is of a ramp type with a rise time $t_{0}$. Then $u(t)$ and $\ddot{u}(t)$ are expressed by $u_{0}(t)$ and $\ddot{u}_{0}(t)$, functions not subject to the rise time, through the following relation:

$$
\begin{aligned}
& u(t)=\frac{1}{t_{0}}\left[u_{0}(t)-u_{0}\left(t-t_{0}\right)\right] \\
& \ddot{u}(t)=\frac{1}{t_{0}}\left[\ddot{u}_{0}(t)-\ddot{u}_{0}\left(t-t_{0}\right)\right] .
\end{aligned}
$$

Displacement $\tilde{u}(t)$ and acceleration $\tilde{\ddot{u}}(t)$ for an arbitrary source time function $D(t)$ are given by the following Duhamel (convolution) integral,

$$
\begin{aligned}
& \tilde{u}(t)=\int_{0}^{t} u_{0}(\tau) \ddot{D}(t-\tau) \mathrm{d} \tau \\
& \ddot{\tilde{u}}(t)=\int_{0}^{t} \ddot{u}_{0}(\tau) \ddot{D}(t-\tau) \mathrm{d} \tau .
\end{aligned}
$$

Illustrations (E) and (F) in Fig. 12 (in these figures, the rise time is fixed at $0.5 \mathrm{sec}$ ) show the accelerations for source time functions which rise suddenly at the start and arrive at the final dislocation value smoothly as designated at the right-hand side of the figure. $(\mathrm{G})$ and $(\mathrm{H})$ are those for the source time functions, the first derivatives of which are continuous. The wave forms presented in Fig. 12 have fewer peaks and do not give a satisfactory peak-topeak correspondence with the main part of the recorded acceleration of Fig. 8. This fact indicates that the source time functions considered here are not proper for this earthquake, suggesting that the simple ramp function is a good approximation for the source time function of this earthquake. The theoretical ground displacements for the cases of (A), (B), (G) and (F) are illustrated in Fig. 13.

\section{Effective Stress and Stress Drop}

Recently, several investigators (BRUNE (1970) and others) developed mathematical models of crack, in order to estimate the effective stress which generates an earthquake. However, the source time function of their models are not of a ramp type and are inconsistent with the result obtained in the previous section. Moreover, OHNAKA (1973) pointed out, on the basis of the 


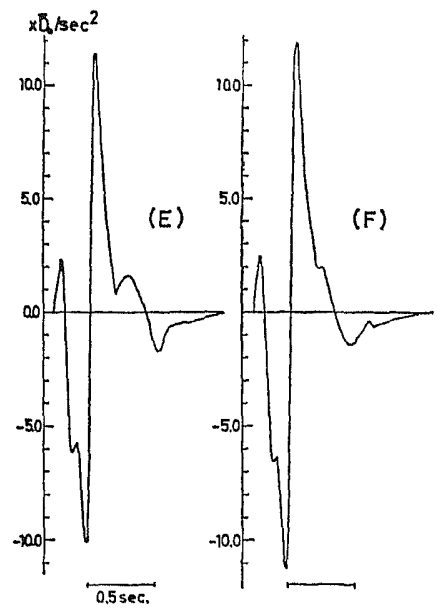

(E)

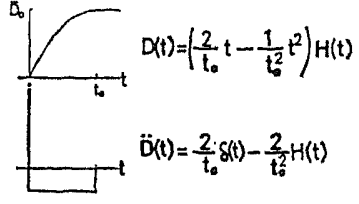

(F)
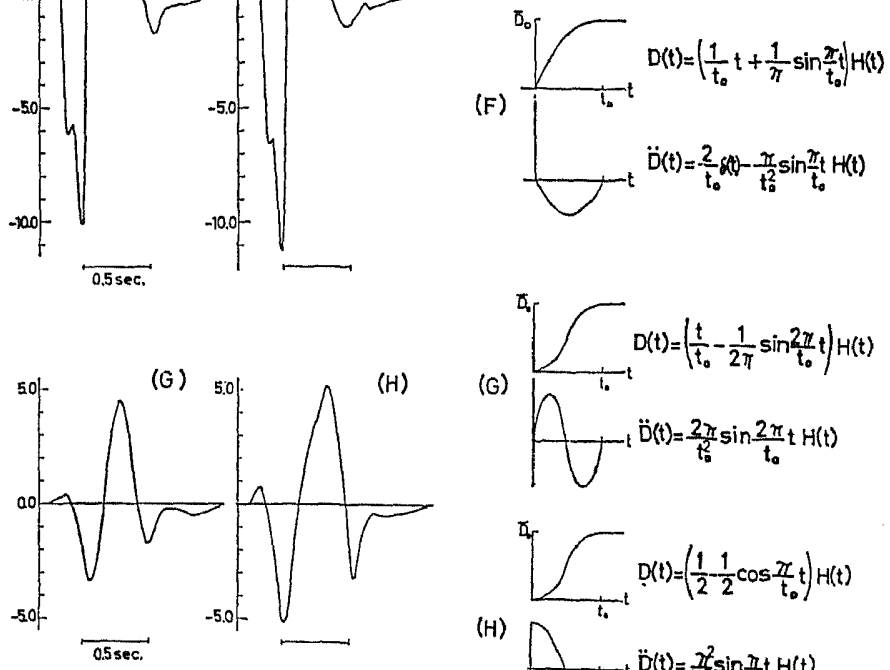

(G)

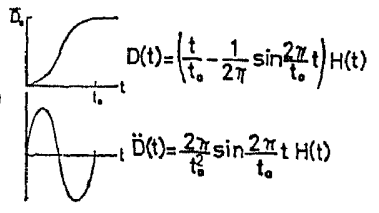

(H)
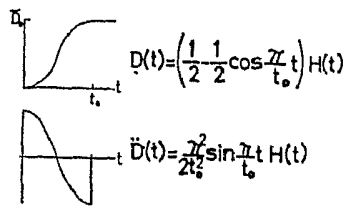

\section{THEORETICAL}

\section{ACCELERATIONS SOURCE TIME FUNCTIONS}

Fig. 12. Theoretical accelerations for various types of source time function. Source time functions and their second derivatives are given at the right-hand side of this figure.

results of laboratory experiments, that mathematical modelings of the crack without consideration of the effect of friction on the rupturing process are invalid. Therefore, although we calculate the value of the effective stress based on their models in this section, it is only a tentative value at this stage.

BRUNE (1970), taking deceleration by the effects of the edge of the dislocation surface into account, gave a simple relation between $\dot{D}(t)$ and $\sigma_{a}$ (effective stress) in the case of instantaneous crack with finite length $2 a$,

$$
\dot{D}(t)=\frac{\sigma_{a} v_{s}}{\mu} \mathrm{e}^{-\left(v_{s} / a\right) t} .
$$

Averaging $\dot{D}(t)$ over a time $a / v_{s}$, we have

$$
\bar{D}=\frac{\sigma_{s} v_{s}}{\mu} \times 0.63 \text {. }
$$




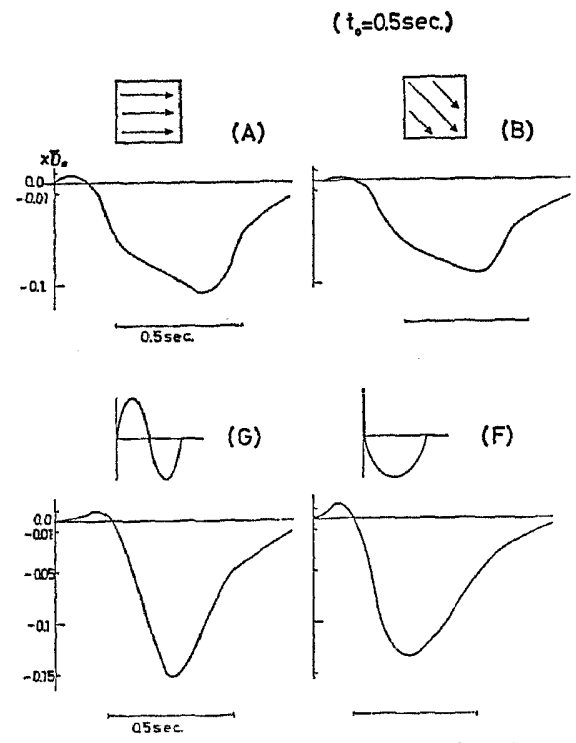

Fig. 13. Theoretical ground displacements for the cases of (A), $(B),(G)$, and $(F)$ in Figs. 10 and 11.

IDA and AKI (1972), considering the conditions that the dislocation distribution over the fault is uniform except near the edges and that excessive stress concentration near the edges disappears, gave the following relation,

$$
\sigma_{e}=\frac{\mu D}{\pi v_{c} t_{0}} \sqrt{1-\left(\frac{v_{c}}{v_{s}}\right)^{2}} .
$$

KANAMORI (1972), studying the deceleration effects of the stopping of the propagating front on the basis of the results of HANSON and SANFORD (1970) and Hamano (personal communication), derived the following relation,

$$
\sigma_{e}=\frac{\mu \bar{D}}{v_{s}}\left(1+\frac{v_{s}}{v_{c}}\right) .
$$

Taking the values, $\mu=2 \times 10^{11} \mathrm{dyne} \cdot \mathrm{cm}^{-2}, v_{s}=3.5 \mathrm{~km} / \mathrm{sec}, v_{c}=2.2 \mathrm{~km} / \mathrm{sec}$, $\bar{D}_{0} /\left(2 \times t_{0}\right)=50 \mathrm{~cm} / \mathrm{sec}$, we have 45 bars, 25 bars and 40 bars from Eqs. (8), (9), and (10), respectively. This leads to the possible range of the effective stress,

$$
\sigma_{e}=25-50 \text { bars. }
$$

From a relation $M_{0}\left(1.4 \times 10^{25} \mathrm{dyne} \cdot \mathrm{cm}\right)=\mu \breve{D}_{0} L W(L=30 \mathrm{~km})$, we get $W=$ $3.5-5 \mathrm{~km}$. From the formula (12) given in the paper of KNOPOFF (1958),

$$
\Delta \sigma=\frac{4 u \breve{D}_{0}}{\pi W}
$$


we get

$$
\Delta \sigma=25-50 \text { bars, }
$$

for the estimate of the stress drop. If we fix $W=10 \mathrm{~km}$ following EAToN et al. (1970), we get

$$
\Delta \sigma=12.5-17.5 \text { bars. }
$$

For the possible range of the stress drop, we get

$$
\Delta \sigma=10-50 \text { bars, }
$$

taking uncertainty of the used value of the parameters into account.

\section{Concluding Remarks}

9-1. The focal parameters determined in the present work are listed in Table 1. However, the values connected with the dislocation estimate may contain an error within a factor of 2 .

Table 1. Source parameters obtained in this study.

\begin{tabular}{cccccc}
\hline$t_{0}$ & $\bar{D}_{0}$ & Depth & Stress drop & $\bar{D}_{0} / 2 t_{0}$ & Effective stress \\
\hline $0.5 \mathrm{sec}$ & $50 \mathrm{~cm}$ & $5.0 \mathrm{~km}$ & $12.5-25 \mathrm{bar}$ & $50 \mathrm{~cm} / \mathrm{sec}$ & $25-50 \mathrm{bar}$ \\
\hline $0.7 \mathrm{sec}$ & $70 \mathrm{~cm}$ & $3.5 \mathrm{~km}$ & $17.5-50 \mathrm{bar}$ & $50 \mathrm{~cm} / \mathrm{sec}$ & $25-50 \mathrm{bar}$ \\
\hline \multicolumn{5}{c}{$\begin{array}{c}t_{0}=0.5-0.7 \mathrm{sec} \\
\bar{D}_{0}=50-70 \mathrm{~cm} \\
\text { Stress drop }=10-50 \mathrm{bar} \\
\text { Effective stress }=25-50 \mathrm{bar}\end{array}$}
\end{tabular}

The average dislocation $\bar{D}_{0}=50-70 \mathrm{~cm}$ seems to be contradictory to the surface fracture of $5-10 \mathrm{~cm}$ reported by BROWN and VEDDER (1967). It should be considered to be due to the soft, thin sediment layer, overlying the basement rock, as AKI (1968) pointed out. The fact that the large recorded acceleration is inexplicable without the large dislocation distribution $\left(D_{\operatorname{mnx}}=\right.$ $60-90 \mathrm{~cm}$ ) at the shallow segment on the fault is rather important.

9-2. The ramp function may be a good approximation for the source time function.

9-3. Parallel (to the fault plane) and vertical components of the theoretical acceleration computed by the focal parameters obtained in this study are presented in Fig. 14. The vertical component does not show a significant resemblance to the recorded one (see Housner and Trifunac, 1967). Since the amplitude of the acceleration of the vertical component is small and comparable with the short-period noise, the main feature of the acceleration controlled directly by the faulting may be hidden in the short-period noise. 

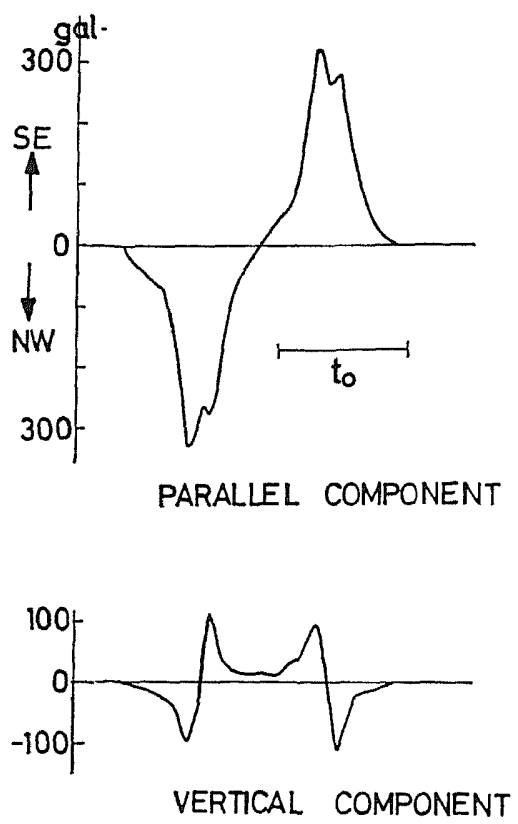

Fig. 14. Theoretical accelerations of parallel and vertical components calculated by the source parameters determined by this study. As $\overline{D_{0}} / t_{0}$ is estimated to be $50 \mathrm{~cm} / \mathrm{sec}$, the calculated amplitudes of the acceleration of these components take the same values irrespective of the rise time estimate.

9-4. We obtain a maximum amplitude of about $7 \mathrm{~cm}$ and a pulse width of $0.5-1.0 \mathrm{sec}$ for the perpendicular component of the ground displacement if we use the values of the source parameters as listed in Table 1. The value of about $7 \mathrm{~cm}$ remarkably disagrees with the estimated value of about $30 \mathrm{~cm}$ of the integrated ground displacement obtained by HousNer and TrIFUNAC (1967) and AKI (1968). If we estimate the average dislocation $\widetilde{D}_{0}$ for this earthquake by this value of about $30 \mathrm{~cm}$, we obtain $\bar{D}_{0}=250-500 \mathrm{~cm}$ as discussed in Section 4. Therefore, the estimated value of about $30 \mathrm{~cm}$ seems to be too large for the perpendicular component of the ground displacement due to this earthquake.

Since the relative magnification of the operated accelerograph at the period 1-2 sec is $0.004-0.001$, the information included in this period range is presumed to be decreased too greatly. Therefore, it is suspected that the short period noise and a constant base line error might be magnified in the numerical integration of the recorded acceleration with respect to time beyond expectation. From these reasons, the integrated ground displacements in this case may not be good approximations for the true ground displacements. 
9-5. The theoretical maximum acceleration $\ddot{u}_{\max }$ and the effective stress $\sigma_{e}$ are approximately proportional to $\bar{D}_{0} / t_{0}$, if a source time function of a ramp type is assumed:

$$
\ddot{u}_{\max } \propto \sigma_{e} \propto \bar{D}_{0} / t_{0} .
$$

Therefore, a source study on the dynamical process in the near-source region is very important from the viewpoint of earthquake engineering.

9-6. The demerit of the direct comparison of the accelerations is that the error caused by the numerical differentiation of the theoretical ground displacements may be large and that the wave forms are easily disturbed by the minor process of rupturing. However, as indicated in the present work, for the acceleration recorded at the Cholame No. 2 station, the above demerit can be eliminated and more extensive information on the dynamical process of faulting can be obtained by direct comparison, though it may include some uncertainty in the dislocation estimate.

9-7. All of the results in this study are derived under the assumption that the seismic waves for the semi-infinite medium are good approximations for those in the multilayered medium partially including trans-sonic rupture propagation. In order to analyse the record more exactly, further progress in the investigation of seismic waves in the multilayered medium as SATO's (1973) is needed.

I wish to express my sincere thanks to Professor Ryosuke Sato, Professor Tomowo Hirasawa and Mr. Yasunori Suzuki for their helpful discussions and critical reading of the manuscript. I am indebted to the Seismological Field Survey for kindly providing us with the accelerogram recorded at the Cholame No. 2 station.

Numerical computations were done by a HITAC 5020E of the Computer Center of the University of Tokyo.

\section{REFERENCES}

Akr, K., Seismic displacements near a fault, J. Geophys. Res., 73, 5359-5376, 1968.

AKI, K., Earthquake mechanism, Tectonophysics, 13, 423-446, 1972.

Brown, R. D. and J.G. VedDer, Surface tectonic fractures along the San Andreas Fault, Geol. Surv. Prof. Paper, 579, 2-23, 1967.

BRUne, J. N., Tectonic stress and the spectra of seismic shear waves from earthquake, $J$. Geophys. Res., 75, 4997-5009, 1970.

Cloud, W. K. and V. Perez, Accelerograms-Parkfield earthquake, Bull. Seism. Soc. Amer., 57, 1179-1192, 1967.

Eaton, J. P., Instrumental seismic studies, Geol. Surv. Prof. Paper, 579, 57-66, 1967.

EAton, J.P., M.E. O'NerL, and J. N. Murdock, Aftershocks of the Parkfield-Cholame, California, earthquake; A detailed study, Bull. Seism. Soc. Amer., 60, 1151-1197, 1970.

Filson, J. and T. McEvilly, Love wave spectra and the determination of the 1966 Parkfieid sequence, Bull. Seism. Soc. Amer., 57, 1245-1257, 1967.

HANSON, M. and A. R. SANFORD, A two-dimensional source function for a dynamic brittle bilateral tensile crack, Bull. Seism. Soc. Amer., 60, 1209-1219, 1970. 
Haskell, N. A., Total energy and energy spectral density of elastic wave radiation from propagating faults, Bull. Seism. Soc. Amer., 54, 1811-1841, 1964.

HASKell, N. A., Elastic displacements in the near-field of a propagating fault, Bull. Seism. Soc. Amer, 59, 865-908, 1969.

Housner, G. W. and M. D. TrifunaC, Analysis of accelerograms-Parkfield earthquake, Bull. Seism. Soc. Amer., 57, 1193-1220, 1967.

IDA, Y. and K. AKI, Seismic source time function of propagating longitudinal-shear cracks, J. Geophys. Res., 77, 2034-2044, 1972.

KANAMORI, H., Seismological evidence for a lithospheric normal faulting. The Sanriku earthquake of 1933, Phys. Earth Planet. Interiors, 4, 289-300, 1971.

KANAMORI, H., Determination of effective tectonic stress associated with earthquake faulting-The Tottori earthquake of 1943, Phys. Earth Planet. Interiors, 5, 426-434, 1972.

KaWASAKI, I., Y. SuzukI, and R. SATo, Seismic waves due to double couple source in a semiinfinite space, Part 1, Zisin, 25, 207-217, 1972 (in Japanese with English summary).

KAWASAkr, I., Y. Suzukr, and R. SAto, Seismic waves due to a shear fault in a semi-infinite medium. Part I, J. Phys. Earth, 21, 251-254, 1973.

KAWASAKI, I, and Y. SUZUKI, Rise time and effective stress estimation from comparison of near-field data with theoretical seismograms in a semi-infinite medium; Sanriku earthquake of March 2, 1933., J. Phys. Earth, 22, 223-236, 1974.

KNopoff, L., Energy release in earthquake, Geophys. J., 1, 44-52, 1958.

Lanczos, C., Applied Analysis, Kinokuniya Bookstore Co., Tokyo, 1956.

Maruyama, T., On the force equivalents of dynamical elastic dislocations with reference to the earthquake mechanism, Bull. Earthq. Res. Inst., 41, 467-486, 1963.

Mrkumo, T., Faulting process of the San Fernando earthquake of February 9, 1971, inferred from static and dynamic near-field displacements, Bull. Seism. Soc. Amer., 63, 249-269, 1973.

OHNAKA, M., Experimental studies of stick-slip and their application to the earthquake source mechanism, J. Phys. Earth, 21, 285-303, 1973.

SATo, R., Formulations of solutions for earthquake source models and some related problems, J. Phys. Earth, 17, 101-110, 1969.

SATo, R., Seismic waves due to a dislocation source model in a multi-layered medium, Part I. Theory, J. Phys. Earth, 21, 155-172, 1973.

Scholz, C. H., M. Wyss, and S. H. SMITH, Seismic and aseismic slip on the San Andreas fault, J. Geophys. Res., 74, 2049-2069, 1969.

TsAI, Y. and K. AKI, Simultaneous determination of the seismic moment and attenuation of seismic surface waves, Bull. Seism. Soc. Amer., 59, 275-287, 1969. 